\title{
Ein Gesamtpaket schnüren
}

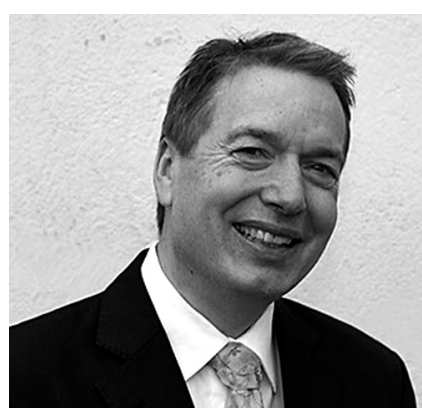

VON MICHAEL STREMLAU Michael Stremlau ist Geschäftsführer des Jobcenters Kiel. Er ist seit 1973 bei der Bundesagentur für Arbeit, davon 20 Jahre in unterschiedlichen Führungsgpositionen. jobcenter-kiel.mitte@jobcenterge.de

\author{
Insbesondere bei der Beschäftigungsförderung für \\ junge Menschen kommt es auf individuell angepasste \\ Maßnahmen an, die sich an der Lebenswirklichkeit von \\ Jugendlichen orientieren. Das Jobcenter Kiel hat als \\ Sozialleistungsträger dazu ein Gesamtkonzept \\ entwickelt und umgesetzt.
}

Es ist zurzeit etwas ruhiger um das Gesetz zur Grundsicherung für Arbeitsuchende (SGB II) geworden. Themen wie der Mindestlohn oder die Rentenreform stehen derzeit im Focus der Öffentlichkeit. Das ist grundsätzlich zu begrüßen, denn wenn man jahrelang die Hitliste der Sozialberichterstattung angeführt hat, tut es gut, wenn man mal in Ruhe arbeiten kann und ausschließlich die Menschen im Mittelpunkt des Handelns stehen. Denn darum geht es: Menschen in schwierigen Lebenssituationen auf den Weg zu bringen. Und am Ende sollen eine Ausbildung oder eine Arbeitsaufnahme stehen, die ein selbstbestimmtes Leben ermöglichen. konstatieren, dass viele den Absprung in Ausbildung oder Arbeit nach dem Schulbesuch nicht geschafft haben und zum Teil schon langfristig von staatlichen Transferleistungen leben.

Das Jobcenter Kiel betreut aktuell 23.144 erwerbsfähige Leistungsberechtigte im Alter von 15 bis 65 Jahren. Darunter sind 4.184 Jugendliche unter 25 Jahre. Etwa die Hälfte von ihnen geht noch zur Schule. Rund 800 befinden sich auf dem Weg in Richtung Ausbildung oder Arbeit: Aber 1.200 sind schon langzeitarbeitslos und versuchen den Anschluss an den Arbeitsmarkt zu finden.

\section{"Langzeitarbeitslose Jugendliche müssen oft zunächst ihr Leben in den Griff bekommen"}

Hört sich einfach an, ist es aber nicht. Denn nicht alle Arbeitsuchenden haben gleichermaßen von der positiven Arbeitsmarktentwicklung der letzten Jahre profitiert. Insbesondere Langzeitarbeitslose haben nach wie vor deutlich schlechtere Chancen eine Arbeit zu finden, als gut qualifizierte Arbeitsuchende. Und damit möchte ich auf eine Gruppe von Langzeitarbeitslosen zu sprechen kommen, die mir besonders am Herzen liegt: Die Jugendlichen unter 25 Jahre. Denn auch bei den Jugendlichen müssen wir
Alle Jugendlichen werden im Jobcenter für Jugendliche betreut, das man durchaus als Vorläufer einer Jugendberufsagentur bezeichnen kann. Denn neben den Integrationsfachkräften ist nicht nur die Berufsberatung im Haus tätig, es gibt auch Werkstätten, in denen Maßnahmen von Bildungsträgern durchgeführt werden. Ergänzt wird das Angebot durch kommunale soziale Begleitmaßnahmen, wie beispielsweise die Schuldnerberatung oder eine psychosoziale Clearingstelle. 


\section{jobcenter.kiel}

\section{Beschäftigungsförderung für}

\section{Ausbildung}

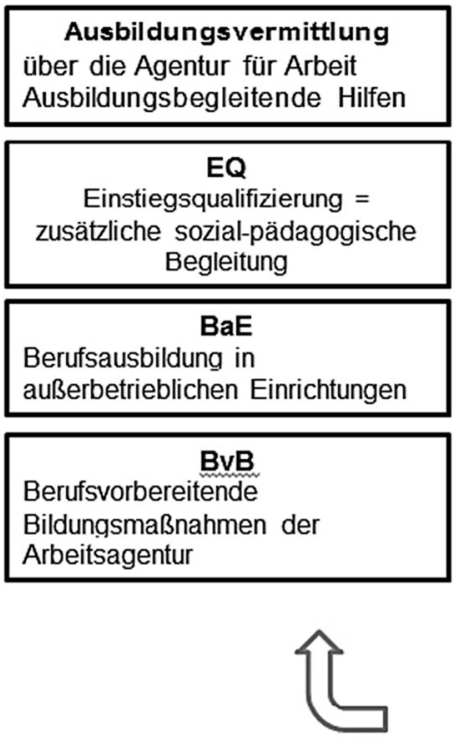

Jugendliche
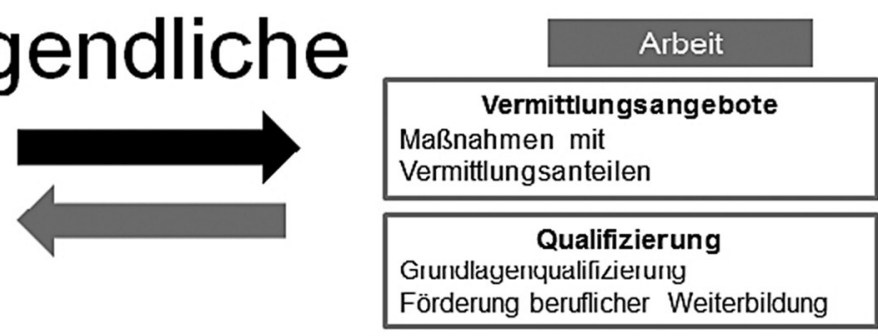

AGH Beschäftigung

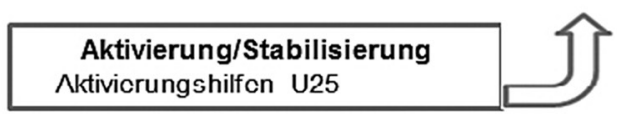

vertiefte Berufsorientierung

Talentschmiede

interkulturelles Bildungsbüro

Schule sonstige Angebote

Kommunale sozlale

Begleitmaßnahmen

psychologische Clearingstelle

Schuldnerberatung

Jugendmigrationsdienst

Mehrgenerationenhaus u.a

Das Jobcenter Kiel hat für die Beschäftigungsförderung von Jugendlichen ein Gesamtkonzept entwickelt, das je nach Vorbildung und Hilfebedarf unterschiedliche Strategien anbietet.

Die Beschäftigungsförderung der Jugendlichen ist vielschichtig. Je nach Vorbildung und Hilfebedarf gibt es unterschiedliche Strategien (vgl. Grafik). Ich möchte den Schwerpunkt auf die 1.200 Jugendlichen legen, die zum Personenkreis der Langzeitarbeitslosen gehören. Diese Jugendlichen brauchen ein komplexes Unterstützungsangebot, um zunächst einmal ihre Lebenssituation in den Griff zu bekommen. Erst wenn der persönliche Bereich weitgehend geordnet ist, kann in der Regel das Thema Ausbildung und Arbeit ernsthaft angegangen werden.

Wir sprechen dabei im Jobcenter Kiel von einem ganzheitlichen Ansatz, in dem nach Möglichkeit auch die gesamte Familie einbezogen werden soll, um die Nachhaltigkeit der verabredeten Maßnahmen sicher zu stellen. Das ist in nur wenigen Fällen möglich, weil viele der
Jugendlichen auf sich allein gestellt sind. Familienverbünde im klassischen Sinne, wie wir sie vielleicht noch alle aus unserer Jugend kennen, erleben viele Jugendliche nicht mehr.

Beispielhaft möchte ich in der Folge Maßnahmen vorstellen, mit denen wir in der Vergangenheit erfolgreich waren. Eines haben sie gemeinsam. Sie sind niederschwellig und ermöglichen Schritt für Schritt den Weg in das Berufsleben.

\section{Was versteht man eigentlich unter einem Jobcenter?}

\section{jobcenter.kiel \\ Arbeitsgemeinschaft für Arbeit und Integration}

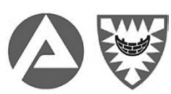

Seit dem 1. Januar 2005 ist das Gesetz zur "Grundsicherung für Arbeitsuchende» in Kraft. Mit der Einführung des Sozialgesetzbuches Zweites Buch (SGB II) wurden die bisherigen Systeme der Arbeitslosenhilfe und Sozialhilfe für Erwerbsfähige zu einer einheitlichen Grundsicherung für Arbeit- suchende zusammen geführt. Vor Ort sorgen die meist aus Kommunen und Bundesagentur für Arbeit gebildeten gemeinsamen Einrichtungen als Jobcenter für die Umsetzung des neuen Gesetzes. Ein Jobcenter ist danach eine juristische Person des öffentlichen Rechts für einen Landkreis oder eine kreisfreie Stadt in Deutschland, die für die Durchführung der Grundsicherung für Arbeitsuchende nach dem SGB II zuständig ist. Aufgabe der Jobcenter ist, Leistungen nach dem SGB II zu gewähren und durch »Fördern und Fordern « den betroffenen Personen die Pers- pektive und Möglichkeit zu eröffnen, ihren Lebensunterhalt künftig aus eigenen Mitteln und Kräften, langfristig und ohne weitere öffentliche Unterstützung zu bestreiten. Mit Jobcenter wird also der zugelassene kommunale Träger (Optionskommune) oder die gemeinsame Einrichtung der Bundesagentur für Arbeit und kommunalem Träger bezeichnet.

Quellen: www.jobcenter-ge.de, www.wikiopedia.org 
Übrigens: Bei der Einrichtung der Maßnahmen wird das Jobcenter von seinem Beirat unterstützt, in dem auch die freien Wohlfahrtsverbände vertreten sind, die gerade in Bezug auf die richtige Herangehensweise bei den Jugendlichen eine wertvolle Hilfe sind.

- Flex-Cafè: Im so genannten FlexCafè arbeiten Jugendliche, die ein grundsätzliches Interesse am gastronomischen Bereich haben. Im Projekt wird kontinuierlich an einer Verbesserung der Tagesstruktur gearbeitet. Weiterhin werden die Jugendlichen in Sachen Schlüsselqualifikationen und Selbstwertgefühl gestärkt. Ein weiterer wesentlicher Punkt ist die Bewältigung von Stress- und Konfliktsituationen. Und es wird zusammen gekocht, um im Mehrgenerationenhaus einen Mittagstisch anbieten zu können. Die direkte Rückmeldung an die Jugendlichen ist sehr dabei wertvoll.

- Kurze Wege: Diese Maßnahme richtet sich an junge Frauen mit einem erhöhtem Beratungs- und Hilfebedarf. Sie sind in der Regel alleinerziehend und suchen den Einstieg in den Arbeitsmarkt. Neben der Erfassung der aktuellen Lebenssituation wird eine verlässliche Kinderbetreuung organisiert, die es den jungen Frauen ermöglicht, zumindest in Teilzeit erste berufliche Perspektiven zu entwickeln.

- 8 + 3: Im Rahmen dieses Projektes werden Jugendliche betreut, die multiple Problemsachverhalte aufweisen und mit ihrer konfliktbeladenen Lebenssituation eigenständig fertig werden müssen. In kleinen Schritten wird Stück für Stück an einer eigenverantwortlichen und gesellschaftlichen Lebensweise gearbeitet. Eine Vielzahl von kommunalen Begleitmaßnahmen, wie die Suchtberatung, die Schuldnerberatung oder psychosoziale Stabilisierungsangebote runden das Gesamtpaket ab. Diese Jugendlichen werden vermutlich Jahre brauchen, um den Anschluss an die Gesellschaft zu finden.
Diese Beispiele zeigen, dass langzeitarbeitslose Jugendliche im Normalfall nicht von heute auf morgen in den Arbeitsmarkt zu integrieren sind. Hier ist Geduld gefragt. Wichtig ist es aus meiner Sicht, gemeinsam mit den Betroffenen einen nachvollziehbaren Plan zu entwickeln, der verstanden und dann gemeinsam umgesetzt wird. So kann man über Zwischenziele die Motivation hochhalten und Erfolge feiern. Für mittel- und langfristige Ziel braucht man aber auch Sicherheit im Haushaltsbereich. Hier ist die Politik gefragt, die Rahmenbedingungen zu verbessern.

Mein Fazit fällt trotz aller Schwierigkeiten bei den Jugendlichen positiv aus. Denn es gelingt jedes Jahr, einige von ihnen auf den Weg zu bringen und es macht viel Freude zu sehen, was sich bei einer entsprechenden Förderung entwickeln kann. Einer von unseren ganz schwierigen Fällen hat gerade als Prüfungsbester seinen Gesellenbrief gemacht. Wenn das kein Ansporn ist unermüdlich weiterzumachen!

Ganz nach unserem Motto: Jeder Jugendliche bekommt jeden Tag eine neue Chance!

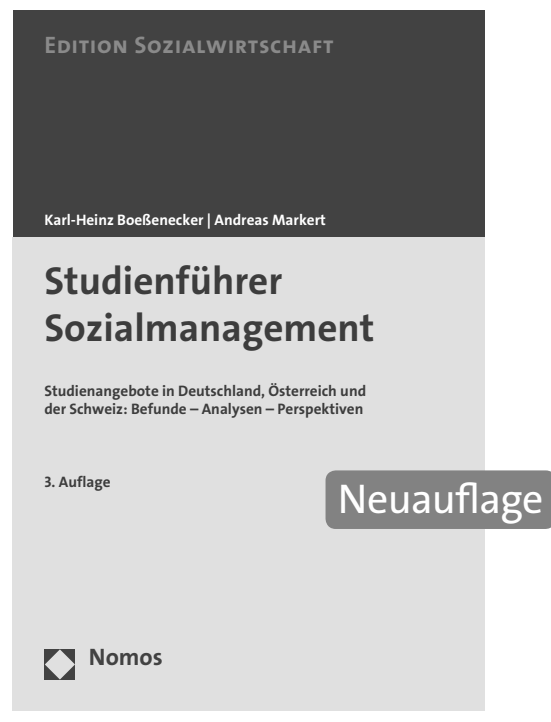

\author{
Studienführer Sozialmanagement \\ Studienangebote in Deutschland, Österreich und der Schweiz: \\ Befunde - Analysen - Perspektiven \\ Von Karl-Heinz Boeßenecker und Andreas Markert \\ 3. Auflage 2014, ca. 200 S., brosch., ca. 19,- $-€$ \\ ISBN 978-3-8487-0956-4 \\ (Edition Sozialwirtschaft, $B d .19)$ \\ Erscheint ca. Juli 2014 \\ www.nomos-shop.de/21806
}

Die Autoren rekonstruieren in der vollständig überarbeiteten und aktualisierten 3. Auflage des Studienführers die an Hochschulen Deutschlands, der Schweiz und Österreichs bestehenden Studiengänge im Bereich Sozialmanagement/Sozialwirtschaft und ordnen diese fachlich ein. Studierende, Lehrende und Praktiker/Praktikerinnen erhalten so eine systematische Übersicht über ein expandierendes Studien- und Handlungsfeld. 